\title{
Elastic Properties of and Dependence on Microstructure of Phosphate Based Bioactive Glasses
}

\author{
V. Rajendran ${ }^{\dagger}$ and A. Nishara Begum \\ Department of Physics, Mepco Schlenk Engineering College, Mepco Engineering College (PO), \\ Virudhunagar (Dt), Tamilnadu-626005, India
}

\author{
Amany A. El-Kheshen and Fatma H. El-Batal \\ Glass Research Department, National Glass Research Centre, Dokki, Cairo, Egypt
}

(Received 31 July 2002; accepted 22 April 2003)

\begin{abstract}
In recent years, bioactive glasses have gained considerable interest among scientists due to their potential applications, such as in the medical field for the replacement of damaged/diseased body parts. One has to optimise the composition of bioactive glasses for effective bonding with bone/dental applications. The preparation of these glasses with different compositions and the characterisation of their physical, chemical and mechanical properties are essential to improve the bioactivity. Ultrasonic non-destructive characterisation of materials is a unique tool used not only for defect characterisation in industry, but also for materials characterisation over a wide range of temperatures. For the present investigation, bioactive glasses in or near the bioactive region, such as $\mathrm{Si}-\mathrm{Al}-\mathrm{K}-\mathrm{Na}-\mathrm{B}-\mathrm{Ce}-\mathrm{Ca}-\mathrm{Ba}-\mathrm{Ti}\left(\mathrm{P}_{2} \mathrm{O}_{5}\right.$ free), Si-Al-K-Li-Na-Zr-P and Si-Al-Na-Ca-Mg-P systems, have been prepared with different compositions by normal melting and annealing techniques. Measurements of ultrasonic velocities, both longitudinal and shear, and of attenuation have been made at room temperature with such bioactive glasses for different thermal treatments operated at a fundamental frequency of $5 \mathrm{MHz}$. Further, frequencydependent ultrasonic velocity and attenuation measurements were made for different frequencies of longitudinal waves. The frequency-dependent attenuation was fitted into a linear relation $\alpha=\beta f^{N}$. The observed increases in density, velocities and elastic constants of bioactive glasses as a function of thermal treatment temperature in the above bioactive glasses are due to an increase in network bonds per unit volume and not due to structural rearrangement/cross-link changes. The observed results have been found to be useful in optimising the composition of bioactive glasses for suitable biomedical applications.
\end{abstract}

${ }^{\dagger}$ Member of the International Institute of Acoustics and Vibration (IIAV)

\section{INTRODUCTION}

The technology of bioactive glasses opens up new possibilities in the natural sciences and medicine in view of their use in a variety of emerging new biomedical applications, such as replacement of damaged/diseased body parts. ${ }^{1-4} \mathrm{~A}$ better understanding of the physical, chemical and mechanical properties of bioactive glasses is required for wider biomedical applications. One can control various properties of bioactive glasses not only by changing their composition but also by subjecting them to different thermal treatments. For the effective bonding of bioactive glass in bone and dental applications, one has to optimise the composition, which requires a complete knowledge of the bone stiffness and mechanical properties. $^{5}$

Earlier studies ${ }^{6-8}$ of bioactive glasses reveal their importance in biomedical applications in view of their high bioactivity and high fracture toughness. This is possibly due to the change in microstructural, mechanical and chemical properties of bioactive glasses that result from changes in composition and by varying thermal treatments. As discussed in our earlier studies, 9,10 even though several techniques, such as static bending and resonance, are available for the evaluation of elastic properties of bioactive glasses, the ultrasonic nondestructive testing (NDT) technique has been found to be more sensitive when exploring the change in elastic and structural properties of bioactive glasses. ${ }^{11,12}$ This is mainly due to the various advantages of the ultrasonic NDT technique in comparison with other techniques, some of which are destructive or semi-destructive. Further, during the ultrasonic testing, the material will not be subjected to any damage. In addition, it is possible to probe into the macro-, microand submicroscopic particles due to the close association of the ultrasonic waves with elastic and inelastic properties of materials, the availability of many modes of vibration and the wide range of frequency selection of the ultrasonic waves. Therefore, in the present investigation, bioactive glasses with and without $\mathrm{P}_{2} \mathrm{O}_{5}$ content have been prepared for different compositions to explore 1) the effect of the frequency of the ultrasonic sound velocity and 2) the effect of thermal treatments on the ultrasonic properties in all bioactive glasses. The observed results are discussed in terms of the changes in structure, stability and elastic properties of bioactive glasses. 